\title{
ON CERTAIN RANDOM POLYGONS OF LARGE AREAS
}

\author{
IGOR N. KOVALENKO \\ STORM UNL ${ }^{1}$ \\ 166-220 Holloway Road \\ London N7 8DB, United Kingdom \\ and \\ V.M. Glushkov Institute of Cybernetics \\ National Academy of Sciences of the Ukraïna
}

(Received April, 1997; Revised January, 1998)

Consider the tesselation of a plane into convex random polygons determined by a unit intensity Poissonian line process. Let $M(A)$ be the ergodic intensity of random polygons with areas exceeding a value $A$. A two-sided asymptotic bound

$$
\exp \left\{-2 \sqrt{A / \pi}+c_{0} A^{1 / 6}\right\}<M(A)<\exp \left\{-2 \sqrt{A / \pi}+c_{1} A^{1 / 6}\right\}
$$

is established for large $A$, where $c_{0}>2.096, c_{1}<6.36$.

Key words: Stochastic Geometry, Random Set(s), Random Tesselation, Point Process(es).

AMS subject classifications: $60 \mathrm{D} 05$.

\section{The Problem Statement and Main Results}

This paper is devoted to the discussion of a problem posed by D.G. Kendall in his foreword to the book [9]. The problem concerns the investigation of the tail of the distribution of the area of a random polygon.

Consider a unit intensity Poissonian line process in $\mathfrak{R}^{2}$. Such a process can be determined by a planar Poissonian process of points $\left(p_{i}, \varphi_{i}\right)$, with a planar intensity $1 / \pi$ in the band $\mathscr{B}_{+} \times(0,2 \pi)$, in such a way that each of the points generates a random line with polar coordinates $\left(p_{i}, \varphi_{i}\right)$ for the foot of the perpendicular from the origin $O$ to the line. Processes of this kind have been investigated thoroughly by Miles [6-8] and others. (Miles uses an equivalent definition: $p_{i} \in \Re_{,}, \varphi_{i} \in(0, \pi)$.) The line processes determines the tesselation of the plane into convex random polygons.

Consider the ergodic intensity $M(A)$ of random polygons with areas exceeding a value $A$.

\footnotetext{
${ }^{1}$ Postal address.
}

Printed in the U.S.A. (C)1998 by North Atlantic Science Publishing Company 
It is necessary to specify a precise definition of ergodic intensities for random polygons. The simplest way to do this is a reduction to the ergodic intensity of an appropriate point process. Evidently, all the sides of a random polygon have different lengths (almost surely). Thus every random polygon $K$ can be associated with a well-defined point $P_{K}$, namely, its vertex such that, when running along the contour of $K$ in the positive direction, the greatest of its sides has $P_{K}$ as its endpoint.

We define the function $M(A)$ as the ergodic intensity of the planar point process of random points $P_{K}$ considering only random polygons $K$ with areas $A(K)>A$. In turn, the ergodic intensity of a planar point process is defined as the mean number of random points in a unit area. From the Korolyuk theorem [3], for a simple stationary point process, the probability that a random point falls into an area element is equivalent to the ergodic intensity of the point process multiplied by the area.

Two asymptotic bounds are established for $M(A)$, as follows.

Theorem 1: The bound

$$
M(A)>\exp \left\{-2 \sqrt{A / \pi}+c_{0} A^{1 / 6}(1+o(1))\right\}
$$

holds for a constant $c_{0}>2.096$ as $A \rightarrow \infty$.

Theorem 2: The bound

$$
M(A)<\exp \left\{-2 \sqrt{A / \pi}+c_{1} A^{1 / 6}(1+o(1))\right\}
$$

holds for a constant $c_{1}<6.36$ as $A \rightarrow \infty$.

\section{Proof of Theorem 1}

Consider an event $\Omega_{r}$ : \{no random line crosses the circle $C_{r}$ of the radius $r$, with the center in the origin $\}$. Evidently, $P\left\{\Omega_{r}\right\}=e^{-2 r}$. If the event $\Omega_{r}$ occurs then the circle $C_{r}$ is surrounded by a random polygon $K_{\varpi}$ (a so-called Crofton cell).

Let $\{(r+X(t), t), 0 \leq t \leq 2 \pi\}$ be the graph of $K_{\varpi}$ in polar coordinates. Then

$$
A\left(K_{\varpi}\right)=\frac{1}{2} \int_{0}^{2 \pi}(r+X(t))^{2} d t .
$$

It is convenient to consider the positive square root $a(K)$ of the area $A(K)$ of a polygon $K$. By the Bounjakowsky (Cauchy) inequality,

$$
2 \pi \int_{0}^{2 \pi} X^{2}(t) d t \geq\left(\int_{0}^{2 \pi} X(t) d t\right)^{2}
$$

Thus equation (3) implies the bound

$$
a\left(K_{\varpi}\right) \geq r \sqrt{\pi} \quad\left(1+\frac{1}{2 \pi r} \int_{0}^{2 \pi} X(t) d t\right)
$$

provided $\Omega_{r}$ occurred.

From Miles' theory, the probability of a random line not crossing a convex figure 
equals $e^{-S / \pi}$, where $S$ is the perimeter of the figure. The event $\Omega_{r} \cap\{X(t)>x\}$ means no crossing by a random line of the convex hull of $C_{r}$ completed by the point $(r+x, t)$. The perimeter of the hull equals $2 r(\pi+\tan \alpha-\alpha)$, where $\alpha=$ $\arccos (r /(r+x))$. Hence,

$$
P\left\{X(t)>x \mid \Omega_{r}\right\}=\exp \{-2 r(\tan \alpha-\alpha) / \pi\} .
$$

It is convenient to introduce a monotonic transformation $\alpha(t)=\arccos (r /(r+$ $X(t))$ ). Since $\{X(t)>x\}=\{\alpha(t)>\alpha\}$, the lefthand side of equation (5) is just $P\left(\alpha(t)>\alpha \mid \Omega_{r}\right\}$. Moreover, consider truncated random variables

$$
\bar{\alpha}(t)=\left\{\begin{array}{cc}
\alpha(t) & \text { if } \alpha(t)<\alpha_{0} \\
0 & \text { if } \alpha(t)>\alpha_{0}
\end{array}\right.
$$

and $\bar{X}(t)=r((1 / \cos \bar{\alpha}(t)-1)$. Thanks to equation (5), the conditional p.d.f. $f(\alpha)$ of the random variable $\bar{\alpha}(t)$ has the form

$$
f(\alpha)=(2 r / \pi) \tan ^{2} \alpha \exp \{-2 r(\tan \alpha-\alpha) / \pi\}
$$

inside the interval $\left(0, \alpha_{0}\right)$, provided $\Omega_{r}$ occurred. Choose $\alpha_{0}$ as $\alpha_{0}=(\ln r / r)^{1 / 3} \ln \ln r$. It can be easily seen that, $\tan \alpha-\alpha \cong \alpha^{3} / 3, \tan \alpha \cong \alpha$ uniformly in the interval $\{0<$ $\left.\alpha<\alpha_{0}\right\}$; hence

$$
E \bar{\alpha}^{m}\left(t \mid \Omega_{r}\right) \cong(2 r / \pi) \int_{0}^{\alpha_{0}} \alpha^{m+2} \exp \left\{-2 r \alpha^{3} /(3 \pi)\right\} d \alpha ; \text { as } r \rightarrow \infty
$$

for any positive $m$. Moreover, the corresponding integral over the interval $\left\{\alpha>\alpha_{0}\right\}$ can be proved to decrease more rapidly than $r^{-N}$ for a given $N$; therefore,

$$
E \bar{\alpha}^{m} \cong(2 r / \pi) \int_{0}^{\infty} \alpha^{m+2} \exp \left\{-2 r \alpha^{3} /(3 \pi)\right\} d \alpha, \text { as } r \rightarrow \infty .
$$

In particular,

$$
\begin{gathered}
E\left\{\alpha^{2}(t) \mid \Omega_{r}\right\} \cong(3 \pi /(2 r))^{2 / 3} \Gamma(5 / 3) \text { as } r \rightarrow \infty \\
E\left\{\alpha^{4}(t) \mid \Omega_{r}\right\} \cong O\left(r^{-4 / 3}\right) \text { as } r \rightarrow \infty
\end{gathered}
$$

As $(1 / \cos z)>1+z^{2} / 2$ for any $z: 0<z<\pi / 2$, we have the inequality

$$
\bar{X}(t)>r \bar{\alpha}^{2}(t) / 2 \text {. }
$$

From Equations (8), (6) and (4),

$$
E\left\{a\left(K_{\varpi}\right) \mid \Omega_{r}\right\} \geq r \sqrt{\pi}\left(1+\frac{1}{2}\left(\frac{3 \pi}{2 r}\right)^{2 / 3} \Gamma(5 / 3)(1+o(1))\right) \text { as } r \rightarrow \infty .
$$

Note that $\operatorname{cov}(\bar{\alpha}(t), \bar{\alpha}(\tau))=0$ as soon as the angle distance between $t$ and $\tau$ exceeds $2 \alpha_{0}$. Applying also equation (7), one obtains the bound

$$
\operatorname{Var}\left\{\int_{0}^{2 \pi} \bar{\alpha}^{2}(t) d t \mid \Omega_{r}\right\}=O\left(r^{-5 / 3}(\ln r)^{1 / 3} \ln \ln r\right) .
$$


Equations (9), (8), (6) and (4) imply the bound

$$
P\left\{a\left(K_{\varpi}\right) \geq r \sqrt{\pi}\left(1+\frac{1}{2}\left(\frac{3 \pi}{2 r}\right)^{2 / 3} \Gamma(5 / 3)(1-\delta)\right) \mid \Omega_{r}\right\} \rightarrow 1 \text { as } r \rightarrow \infty
$$

for any $\delta>0$.

Choose $r$ as follows:

$$
r=\frac{a}{\sqrt{\pi}}\left(1-C a^{-2 / 3}(1-2 \delta)\right)
$$

where

$$
C=\pi(3 / 2)^{2 / 3} \Gamma(5 / 3) / 2
$$

Then equation (10) implies the bound

and hence

$$
P\left\{a\left(K_{\varpi}\right)>a \mid \Omega_{r}\right\} \rightarrow 1 \text { as } a \rightarrow \infty
$$

$$
P\left\{\Omega_{r} \cap\left\{a\left(K_{\varpi}\right)>a\right\}\right\} \cong P\left\{\Omega_{r}\right\}=e^{-2 r} .
$$

Inserting $r$, defined by equation (11), into equation (14), and changing $a^{2}$ to $A$ leads to the following bound:

$$
P\left\{C_{r} \subset K_{\varpi} ; A\left(K_{\varpi}\right)>A\right\}>\exp \left\{-2 \sqrt{A / \pi}+c_{0} A^{1 / 6}(1+o(1))\right\}
$$

with $c_{0}=(3 / 2)^{2 / 3} \sqrt{\pi} \Gamma(5 / 3)>2.096$.

To make a passage from probabilities to ergodic intensities, we will prove that a typical Crofton cell lies inside a certain circle of the radius $3 r$, provided the event $\Omega_{r}$ occurred. Consider three concentric circles $C_{r}, C_{2 r}, C_{3 r}$ of the radii $r, 2 r, 3 r$, respectively. The event $\Omega_{r} \cap\{X(t)>2 r\}$ implies the event $\Omega_{r} \cap\left\{X(\tau)>r, \tau \in \Delta_{t}\right\}$ where $\Delta_{t}$ is an interval of a positive length. If $N$ is large enough then

$$
\Omega_{r} \cap\left\{X(\tau)>r, \tau \in \Delta_{t}\right\} \subset \bigcup_{k=0}^{N-1} \Omega_{r} \cap\{X(2 \pi k / N)>r\}
$$

for every $t, 0 \leq t \leq 2 \pi$. Therefore, due to equation (5) with $\alpha=\pi / 3$,

$$
P\left\{\max _{t} X(t)>2 r \mid \Omega_{r}\right\} \leq N \exp \{-2 r(\sqrt{3}-\pi / 3) / \pi\}=o(1) \text { as } r \rightarrow \infty .
$$

We have the relation

$$
\Omega_{r} \cap\left\{\max _{t} X(t) \leq 2 r\right\} \subset \Omega_{r} \cap\left\{P_{K_{\varpi}} \in C_{3 r}\right\}
$$

since

we have

$$
A\left(C_{3 r}\right)=9 \pi r^{2}
$$

$$
9 \pi r^{2} M(A) \geq P\left\{\Omega_{r} \cap\left\{\max _{t} X(t) \leq 2 r\right\}\right\} \cong 9 \pi r^{2} P\left\{\Omega_{r}\right\} .
$$

Equations (11-15) imply the relation in equation (1). 


\section{A Bound for Ergodic Intensities $M_{n}(A)$}

Evidently,

$$
M(A)=\sum_{n=3}^{\infty} M_{n}(A),
$$

where $M_{n}(A)$ is the contribution of $n$-gons to $M(A)$. The following lemma presents an upper bound for $M_{n}(A)$; we set $A=a^{2}$.

Lemma 1: $A$ bound

$$
M_{n}\left(a^{2}\right) \leq \frac{c n}{(2 n-3) ! a}(2 \pi)^{2 n} L_{n-1}
$$

holds true with

$$
L_{n-1}=\int_{y_{i}>0, y_{1}+\ldots+y_{n}>2 a\left(1+\Delta_{n}\right) / \sqrt{\pi}} \exp \left\{-\left(y_{1}+\ldots+y_{n}\right)\right\} d y_{1} \ldots d y_{n-1}
$$

where $y_{n}$ is a function of the remaining $y_{i}$ 's;

$$
\begin{gathered}
y_{n}>\left(y_{1}+\ldots+y_{n-1}\right) /(n-1) \\
\Delta_{n}=\left(\frac{n}{\pi} \tan \frac{\pi}{n}\right)^{1 / 2}-1
\end{gathered}
$$

Proof: Let $K$ be a random $n$-gon such that its vertex $P_{K}$ (see Section 1 for a definition) falls into the circle $C_{\rho}$ of a small radius $\rho$, with center at the origin. Denote vertices of $K$ by $P_{1}, \ldots, P_{n}$ in the positive direction; for definiteness, set $P_{n}=P_{K}$ (i.e., the endpoint of the side of the greatest length). Also, denote the exterior angle of $K$ corresponding to the vertex $P_{i}$ by $\theta_{i}$, and set $x_{1}=\left|P_{n} P_{1}\right| ; x_{i}=P_{i-1} P_{i} \mid$, $2 \leq i \leq n$. For simplicity, we will also give the line $P_{i-1} P_{i}$ the name $X_{i}$. An $n$-gon $K$ can be coded as $(\boldsymbol{\theta}, \boldsymbol{x})$, where $\boldsymbol{\theta}=\left(\theta_{1}, \ldots, \theta_{n-2}\right)$ and $\boldsymbol{x}=\left(x_{1}, \ldots, x_{n-1}\right)$. Given a position of the line $X_{1}$, these parameters determine $x_{n}$ and $\theta_{n-1}$ uniquely as $\rho \rightarrow 0$. For example, $x_{n}=\left|O P_{n-1}\right|$ in the limit. By definition, see Section 1, $x_{n}=$ $\max _{1 \leq i \leq n}\left\{x_{i}\right\}$. It is well known that the perimeter of an $n$-gon $K$ does not exceed the perimeter of a regular $n$-gon, given a value of the area. Thus, $x_{1}+\ldots+x_{n}>$ $2 a \sqrt{\pi}\left(1+\Delta_{n}\right)$ as soon as $A(K) \geq a^{2}$. These notes explain the bounds for $y_{i}$ in equations (17-19), where $y_{i}=x_{i} / \pi$. Consider the probability of the occurrence of a random polygon in elementary volumes $d \boldsymbol{\theta}, d \boldsymbol{x}$, and $\pi \rho^{2}$ for parameters, $\boldsymbol{\theta}, \boldsymbol{x}$, and $P_{K}$, respectively. This differential probability can be considered as the product of the following expressions:

(i) $2 \rho=$ the probability of a random line $X_{1}$ crossing $C_{\rho}$;

(ii) $(2 \pi)^{-n+2} d \theta_{1} \ldots d \theta_{n-2}=$ the probability of the choice of the directions of random lines $X_{2}, \ldots, X_{n-1}$;

(iii) $2^{n-2} \sin \theta_{1} d x_{1} \ldots \sin \theta_{n-2} d x_{n-2}=$ the probability of the crossing of $X_{i}$ by $X_{i+1}$ in the intervals $d x_{i}$, given $\theta_{i}$;

(iv) $2 \rho \sin \theta_{n-1} d x_{n-1} /\left(\pi x_{n}\right)=$ the probability of the occurrence of a random line crossing both an interval $d x_{n-1}$ of the line $X_{n-1}$ and the circle $C_{\rho}$; and

(v) $\exp \left\{-\left(x_{1}+\ldots+x_{n}\right) / \pi\right\}=$ the probability of no random line crossing the polygon $K$. [The above formulation is close to that of Miles [6-8]]. 
Applying a usual principle

$$
\int f(\boldsymbol{\theta}, \boldsymbol{x}) d \boldsymbol{\theta} d \boldsymbol{x} \leq \int d \boldsymbol{\theta} \int \sup _{\boldsymbol{\theta}} f(\boldsymbol{\theta}, \boldsymbol{x}) d \boldsymbol{x}
$$

we may integrate in $\boldsymbol{\theta}$ separately:

$$
\begin{gathered}
\int \ldots \int \sin \theta_{1} \ldots \sin \theta_{n-1}\left(d \theta_{1} \ldots d \theta_{n-2}\right) \\
<\int \cdots \int \theta_{1} \ldots \theta_{n-2}\left(2 \pi-\theta_{1}-\ldots-\theta_{n-2}\right) d \theta_{1} \ldots d \theta_{n-2} \\
=(2 \pi)^{2 n-3} /(2 n-3) !
\end{gathered}
$$

Having collected expressions $(i)$ to $(v)$, the bound in equation (20), applying the inequality $x_{n}>\left(x_{1}+\ldots+x_{n}\right) / n>2 a \sqrt{\pi} / n$, and also having divided by $\pi \rho^{2}$, one obtains the bound in equations (16) and (17) directly.

\section{A Proof of Theorem 2}

Let $L_{n-1}^{0}$ denote the contribution of the domain $\left\{y_{1}+\ldots+y_{n-1}<2 a\left(1+\Delta_{n}\right) / \sqrt{\pi}\right\}$ to the integral $L_{n-1}$ [see equation (17)]; $L_{n-1}^{1}=L_{n-1}-L_{n-1}^{0}$. Due to equation (16), we have

$$
M_{n}\left(a^{2}\right) \leq M_{n}^{0}\left(a^{2}\right)+M_{n}^{1}\left(a^{2}\right)
$$

where $M_{n}^{j}\left(a^{2}\right)$ is defined as the righthand side of equation (16) with $L_{n-1}$ changed by $L_{n-1}^{j}(j=0,1)$. We have

$$
\begin{array}{r}
L_{n-1}^{0}<\exp \left\{-2 a\left(1+\Delta_{n}\right) / \sqrt{\pi}\right\} \quad \int \cdots \int d y_{1} \ldots d y_{n-1} \\
y_{i}>0, y_{1}+\ldots+y_{n-1}<2 a\left(1+\Delta_{n}\right) / \sqrt{\pi}
\end{array}
$$

Equation (21) implies the bound $\sum_{n=3}^{N} M_{n}^{0}\left(a^{2}\right)=O(-2 a(1+\varepsilon) / \sqrt{\pi})$ as $a \rightarrow \infty$ for a given $N$; thus, only the case of large $n$ should be investigated. Applying Stirling's formula to the factorials and also applying the relation

one obtains the bound

$$
\Delta_{n} \cong \pi^{2} /\left(6 n^{2}\right)=o(1 / n)
$$

$$
\left.M_{n}^{0}\left(a^{2}\right)<c_{1}\left(n^{4} / a^{2}\right)\left(2 \pi^{3 / 2} e^{3} a / n^{3}\right)^{n} \exp \left\{-2 a\left(1+\pi^{2} /\left(6 n^{2}\right)\right) / \sqrt{\pi} n^{2}\right)\right\} .
$$

To apply a usual asymptotic analysis, see Dingle [2], introduce a variable

$$
x=n /\left(2 \pi^{3 / 2} e^{3} a\right)^{1 / 3}
$$


and search the maximum of the expression

$$
\begin{gathered}
\ln \left(\exp \left\{-2 a \Delta_{n} / \sqrt{\pi}\right\} / x^{3 n}\right) \\
\approx\left\{-18.211 x \ln x-0.0504 x^{-2}\right\} a^{1 / 3} .
\end{gathered}
$$

As the result of computations, the value 6.36 is obtained as an upper bound. By a standard argument in Dingle [2] it can be shown that

for large $a$.

$$
M^{0}\left(a^{2}\right)<\exp \left\{-2 a / \sqrt{\pi}+6.36 a^{1 / 3}\right\}
$$

As for $M_{n}^{1}\left(a^{2}\right)$, consider two cases:

(i) $\quad n \geq a / \ln a$.

(ii) $n<a / \ln a$.

In the case $(i)$ it is sufficient to note that $L_{n-1}^{1}<1$ whereas

$$
\sum_{n} \frac{n}{(2 n-3) !}(2 \pi)^{2 n}=O\left(e^{-2(1-\delta) a}\right) \text { as } a \rightarrow \infty
$$

for a given $\delta>0$, and thus

$$
\sum_{n} M_{n}^{1}\left(a^{2}\right)<\exp \{-2 a / \sqrt{\pi}\}
$$

for large $a$. In case $(i i)$, we omit the factor $\left(1+\Delta_{n}\right)$ in equation (17) and note that

$$
\int_{y_{i}>0, y_{1}+\ldots+y_{n}>x} \ldots \int^{-\left(y_{1}+\ldots+y_{n}\right)} d y_{1} \ldots d y_{n}<(e x / n)^{n} e^{-x}
$$

as soon as $n<x$. Hence,

$$
\begin{gathered}
M_{n+1}^{1}\left(a^{2}\right)<\frac{c(n+1)}{a(2 n-1) !}\left(\frac{2 a e}{\sqrt{\pi} n}\right)^{n} \\
\times(2 \pi)^{2 n+2} \exp \left\{-2 a(1+1 / n) / \pi^{1 / 2}\right\}=Q_{n}(a), \text { say. }
\end{gathered}
$$

From equation (24), we have a relation

$$
Q_{n+1}(a) / Q_{n}(a) \cong 2 a \pi^{3 / 2} n^{-3} \exp \left\{2 a(1+o(1)) /\left(n^{2} \pi^{1 / 2}\right)\right\}
$$

for large $n$. The righthand side of equation (25) is large as $n<a^{(1-\varepsilon) / 2}$ and small as $n>a^{192}$ Hence $\arg \max Q_{n}(a)=a^{\theta}$ where $(1-\varepsilon) / 2<\theta<1 / 2$. For such $n$, equation (24) implies the relation

hence,

$$
M_{n+1}\left(a^{2}\right)<\exp \left\{-2\left(a+a^{1 / 2}\right) / \pi^{1 / 2}\right\}
$$

$$
\Sigma_{n<a / \ln a} M_{n+1}^{1}\left(a^{2}\right)<\exp \left\{-2 a / \pi^{1 / 2}\right\} .
$$

Combining the bounds in equations (22), (23) and (26) leads to the desired equation (2). 


\section{Remarks}

The problem considered here is closely related to a "long-standing conjecture of D.G. Kendall" concerning shapes of random polygons. In a version suggested by Miles [8], this conjecture is as follows: Let $\mu(A) d A$ be the ergodic intensity of random polygons of the type considered as above, and $\mu_{\varepsilon}(A) d A$ be the ergodic intensity of those contours which, moreover, are surrounded by concentric circles of radii $\sqrt{A / \pi}(1 \pm \varepsilon)$. Then:

$$
\mu_{\varepsilon}(A) / \mu(A) \rightarrow 1 \text { as } A \rightarrow \infty
$$

for a given $\varepsilon>0$. For two different proofs of equation (27), both based on an inequality of Bonnesen [1], see Kovalenko [4, 5].

\section{Acknowledgements}

It is my pleasant duty to thank Professor D.G. Kendall for his support of my research in this area, and also Professors R. Gilchrist and L. Takács for their helpful suggestions. I am also very much indebted to my colleague Dr. M.N. Savchuk who has checked the validity of equations throughout the text of the paper.

\section{References}

[1] Bonnesen, T., Über eine Verscharfung der isoperimetrischen Ungleichheit des Kreises in der Ebene und auf der Kugelobertlache nebst einer Anwendung auf eine Minkowskische Ungleichheit für konvexe Korper, Math. Ann. 84 (1921), 216-227.

[2] Dingle, R.B., Asymptotic Expansions: Their Derivation and Interpretation, Academic Press, London and New York 1973.

[3] Franken, P., König, D., Arndt, U. and Schmidt, V., Queues and Point Processes, J. Wiley and Sons, New York 1982.

[4] Kovalenko, I.N., An alternative approach to R.E. Mile's proof of a conjecture of D.G. Kendall concerning the shapes of random polygons, Doc. No. 62810, STORM, Univ. of North London 1997.

[5] Kovalenko, I.N., A proof of the conjecture of David G. Kendall concerning a form of random polygons of large areas, Kibernetika $i$ Sistemnyi Analiz 4 (1997), 3-10. (In Russian).

[6] Miles, R.E., Random polygons determined by random lines in a plane, Proc. of the National Academy of Sciences (USA) 52 (1964), 901-907, 1157-1160.

[7] Miles, R.E., The various aggregates of random polygons determined by random lines in a plane, Adv. Math. 10 (1973), 256-290.

[8] Miles, R.E., A heuristic proof of a long-standing conjecture of D.G. Kendall concerning the shapes of certain large random polygons, Adv. Appl. Prob. (SGSA) 27 (1995), 397-417.

[9] Stoyan, D., Kendall, W.S., Mecke, J., Stochastic Geometry and Its Applications: With a Foreword of D.G. Kendall, J. Wiley and Sons, New York 1987. 


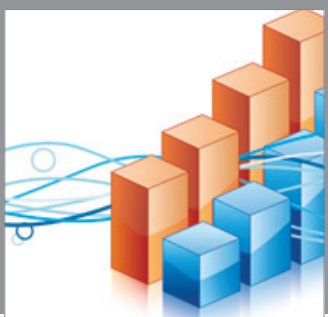

Advances in

Operations Research

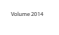

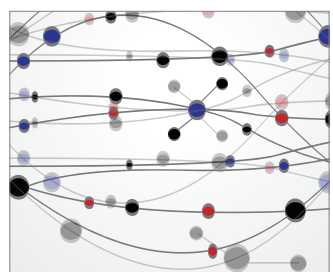

\section{The Scientific} World Journal
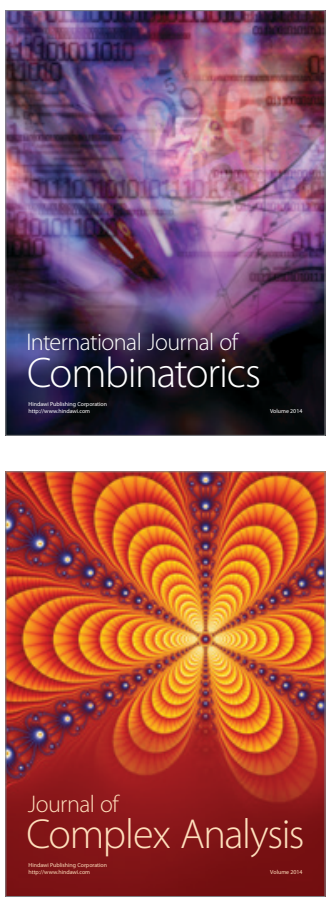

International Journal of

Mathematics and

Mathematical

Sciences
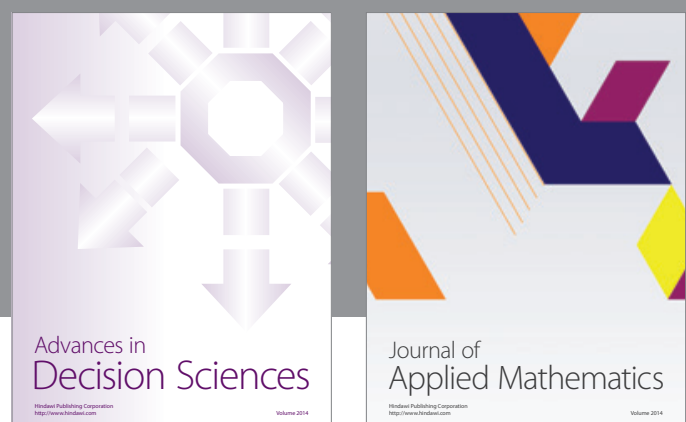

Journal of

Applied Mathematics
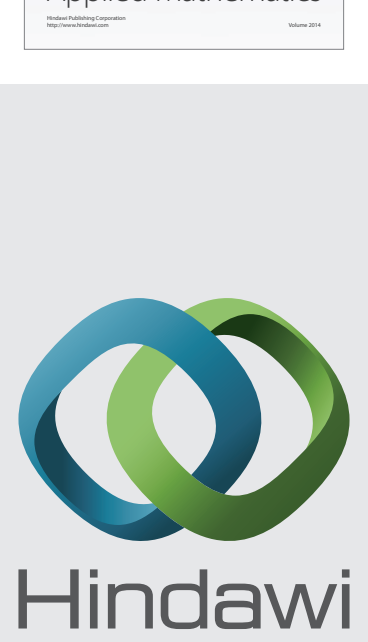

Submit your manuscripts at http://www.hindawi.com
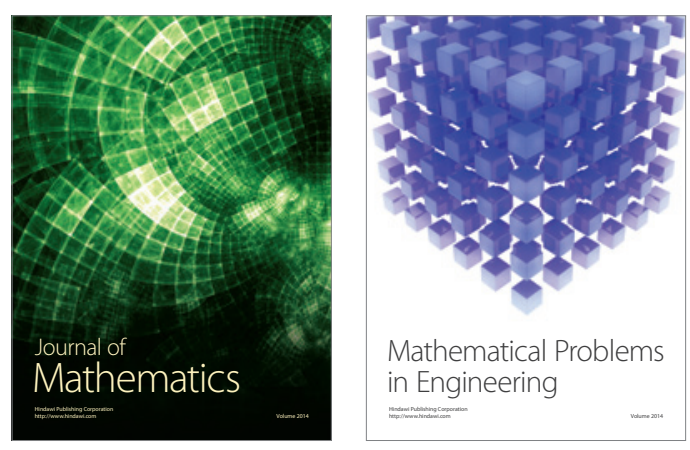

Mathematical Problems in Engineering
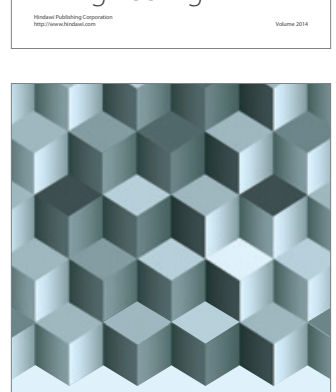

Journal of

Function Spaces
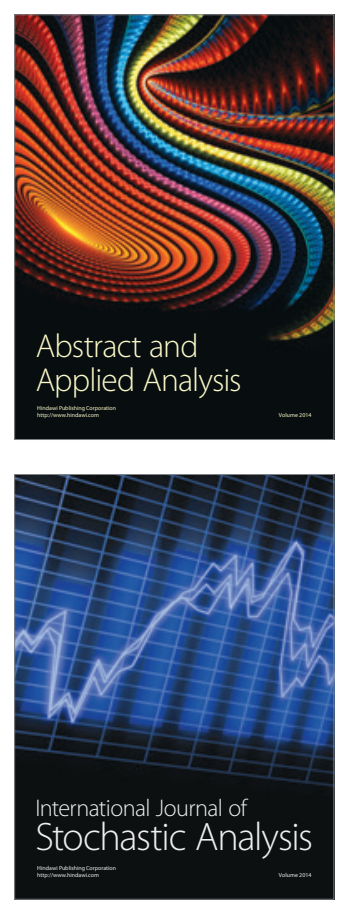

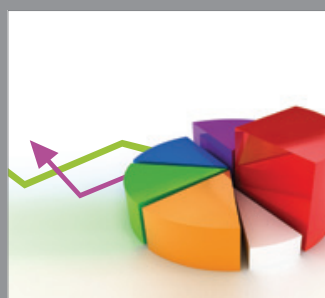

ournal of

Probability and Statistics

Promensencen
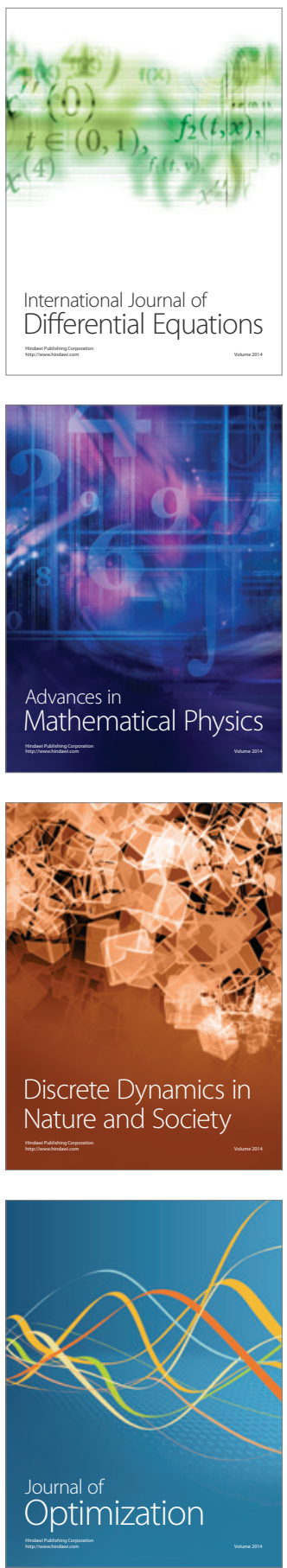